\title{
Asociación entre síndrome de caídas y síntomas depresivos en adultos mayores de once comunidades altoandinas del Perú 2013-2017.
}

\author{
Association between falls and depressive symptoms among elderly people from eleven Peruvian Andean \\ highland communities 2013-2017.
}

Lucía Casahuaman-Orellana ${ }^{1}$, Fernando M. Runzer-Colmenares ${ }^{2,3}$, José F. Parodi ${ }^{3}$

RESUMEN

Objetivo: Determinar si existe asociación entre el síndrome de caídas y la presencia de sintomatología depresiva en adultos mayores de once comunidades altoandinas, entre los años 2013 al 2017. Material y métodos: Estudio retrospectivo, analítico realizado en una muestra de pobladores de 60 años o más residentes de las siguientes localidades: La Jalca, Leimebamba (Amazonas), Llupa, San Pedro de Chaná, Atipayan (Áncash), Pampamarca (Huánuco), Ayahuanco (Ayacucho), Paucarcolla (Puno), Vilca (Huancavelica), Viñac (Lima) y Chacapampa (Huancayo). Se utilizó un instrumento basado en datos generales del paciente, antecedentes patológicos de importancia y examen físico. Resultados: Se entrevistó un total de 411 participantes; $65,94 \%$ de los cuales eran de sexo femenino y $65,45 \%$ vivían en una zona rural. Un 35,23\% del total de caídas ocurrió a consumidores de alcohol; se comprobó un menor promedio de rendimiento físico y de alcance funcional en pacientes con historia de caídas frecuentes y un 48,48\% de los mismos tenían sintomatología depresiva. Conclusiones: Los pacientes con síntomas depresivos tienen 1,62 veces más posibilidades de sufrir caídas en su actividad cuotidiana.

PALABRAS CLAVE: Depresión, caídas, adulto mayor, Andes.

\section{SUMMARY}

Objective: To determine the association between falls and the presence of depressive symptoms in older adults of eleven highland Andean communities between 2013 and 2017. Material and methods: A retrospective, analytical study was conducted in a sample of residents aged 60 years and over, living in the following localities: La Jalca, Leimebamba (Amazonas), Llupa, San Pedro de Chaná, Atipayan (Ancash), Pampamarca (Huánuco), Ayahuanco (Ayacucho), Paucarcolla (Puno), Vilca (Huancavelica), Viñac (Lima) and Chacapampa (Huancayo). An instrument, based on general patient data, important pathological antecedents and physical examination, was utilized. Results: A total of 411 patients were interviewed, $65.94 \%$ of whom were female, and $65.45 \%$ lived in a rural area. Alcohol users accounted for $35.23 \%$ of total falls; a lower average physical performance and functional range were detected in patients who experienced falls, and $48.48 \%$ of the same had depressive symptoms. Conclusions: Patients with depressive symptoms have a 1.62 times higher chance to suffer a fall in their daily activity.

KEYWORDS: Depression, falls, elderly, Andes.

Universidad Científica del Sur. Lima, Perú.

2 Universidad Científica del Sur, Facultad de Ciencias de la Salud, Carrera de Medicina Humana. Lima, Perú.

3 Universidad de San Martin de Porres, Facultad de Medicina Humana, Centro de Investigación del Envejecimiento (CIEN). Lima, Perú. 


\section{INTRODUCCIÓN}

Los trastornos del equilibrio y las caídas en adultos mayores son un problema de salud pública importante, en aumento en la última década (1). Las caídas, además de constituir uno de los síndromes geriátricos más importantes (ya que presentan una alta incidencia y elevada morbimortalidad), generan diversas consecuencias en las personas que las sufren, como fracturas, heridas de importancia, temor a caer, aislamiento social, dependencia en las actividades de la vida diaria (generando una peor calidad de vida) y depresión (2). La depresión, por otro lado, es una enfermedad bastante común en los adultos mayores, que afecta alrededor del $14 \%$ de los mismos (aun cuando con frecuencia está infradiagnosticada). A su vez multiplica las visitas ambulatorias y el gasto sanitario por potenciar el efecto negativo de otras comorbilidades (3).

En México se realizó un estudió en el cual se buscaba asociar la presencia de caídas con algunos factores de riesgo y con la calidad de vida relacionada con la salud, encontrándose que factores como el equilibrio, estado de depresión, confusión, desorientación, mareos, y el uso de diversos medicamentos tienen una asociación con significancia con el diagnóstico de caídas (2).

Según el Instituto Nacional de Estadística e Informática (INEI), para el año 2018 hubo un aumento de la población adulta mayor (considerada a partir de los 60 años de edad) en el país, siendo un total del $10,4 \%$, habiendo una persona adulta mayor en al menos el $41,1 \%$ de los hogares peruanos de zonas rurales (4).

La asociación entre caídas y depresión ha sido explorada en diversos estudios, sin embargo, la información sobre dicho fenómeno en población adulta mayor residente en altura es escasa. Dadas las características sociales, sanitarias y físicas de dicha población, el objetivo del presente trabajo fue encontrar mediante un estudio analítico la asociación entre sintomatología depresiva y el síndrome de caídas en adultos mayores residentes de 11 comunidades altoandinas del país.

\section{MATERIAL Y MÉTODOS}

El estudio actual es un análisis secundario de base de datos. El diseño del estudio primario es tipo analítico. Para el estudio primario, se procedió a recolectar los datos de pobladores de 11 comunidades alto-andinas del Perú, ubicadas en los departamentos de Ancash, Ayacucho, Amazonas, Huancavelica,
Huánuco Huancayo, Lima y Puno durante los años 2013 al 2017: a) Atipayán ubicado a 3364 m s.n.m. (Ancash) b) Llupa a 3511 m s.n.m. (Áncash) c) San Pedro de Chaná ubicado a 3413 m s.n.m. (Ancash) d) Ayahuanco ubicado a 3414 m s.n.m. (Ayacucho) e) La Jalca ubicado a $2800 \mathrm{~m}$ s.n.m. (Amazonas) f) Leimebamba ubicado a $2158 \mathrm{~m}$ s.n.m. (Amazonas) g) Vilca ubicado a 3275 m s.n.m. (Huancavelica) h) Pampamarca ubicado a $3445 \mathrm{~m}$ s.n.m. (Huánuco) i) Paucarcolla ubicado a 3847 m s.n.m. (Puno) j) Viñac ubicado a 3315 m s.n.m. (Lima)k)Chacapampa ubicado a 3358 m s.n.m. (Huancayo). Estas comunidades, se encuentran en la región Sierra de Perú (5).

Criterios de inclusión: participantes de 60 años o más, que hayan firmado el consentimiento informado, atendidos en niveles asistenciales ambulatorios de las 11 comunidades altoandinas seleccionadas.

Criterios de exclusión: Pacientes que se encontraban hospitalizados, pacientes con dificultades para acudir a control ambulatorio, pacientes que no pudieron completar la encuesta, con deterioro auditivo o visual que impedía la realización de las pruebas.

Del total de pobladores mayores de 60 años que cumplían con los criterios de inclusión mencionados (un total de 413 personas), se procedió a excluir a 1 adulto mayor debido a que no se obtuvieron datos del mismo acerca del test de Yesavage, y a un poblador más debido a que no se encontró datos sobre su historial de caídas, dato importante para este estudio. Por lo que finalmente, se analizó datos de un total de 411 participantes.

Para la recolección de datos, se usó un instrumento el cual estaba dividido en 3 partes:

1) Datos generales del paciente: para lo cual se realizó una entrevista con el paciente obteniéndose datos como el sexo, edad (la cual fue separada en tres grupos: de 60 a 70 años, de 71 a 80 años y más de 81 años), estado civil (soltero, casado viudo, divorciado/separado), lugar de vivienda (siendo rural o urbana) si la persona vive acompañada o sola y grado de instrucción (analfabeta/colegio incompleto, colegio completo, técnico superior). Todos estos datos se corroboraron posteriormente con el documento nacional de identidad (DNI). Así mismo se preguntó por como el paciente veía su condición de salud actual (dividiéndose entre excelente, muy bueno, bueno, regular y malo) 
Asociación entre síndrome de caídas y síntomas depresivos en adultos mayores de once comunidades altoandinas.

2) Antecedentes patológicos de importancia: siguiendo con la entrevista se averiguó mediante autoreporte o pregunta al acompañante por consumo de diferentes medicamentos y polifarmacia (considerándose positivo a polifarmacia el uso de 5 medicamentos o más diariamente), historia de caídas (en los últimos 12 meses), presencia de comorbilidades (en este caso, se obtuvo mediante autoreporte o reporte del acompañante datos acerca de enfermedades de base como diabetes mellitus II, enfermedad pulmonar obstructiva crónica e hipertensión arterial, para averiguar por problemas de visión se hizo la siguiente pregunta: “¿Tiene problemas de visión que limitan su vida diaria?, para problemas de audición se hizo la siguiente pregunta: " ¿Tiene problemas de audición que limitan su vida diaria?" y para incontinencia urinaria se usó la siguiente pregunta: ¿Tiene problemas perdiendo el control de la continencia urinaria cuando no desea orinar?. En base a estas seis comorbilidades se procedió a agrupar en 3 grupos: ninguna, una comorbilidad o 2 o más comorbilidades). Además, se consignó el consumo de alcohol, cigarros y consumo de hoja de coca. También, se realizó la siguiente pregunta " $¿$ Se siente frecuentemente triste $o$ deprimido?" para explorar frecuencia de síntomas depresivos.

3) Examen físico: Para esta parte del instrumento se usaron como referencia diferentes escalas:

Escala de depresión geriátrica de Yesavage (6): se empleó una versión ultra corta del test de 5 preguntas “¿Está básicamente satisfecho con su vida?, ¿Se siente a menudo aburrido?, ¿Se siente a menudo sin esperanza?, ¿Prefiere quedarse en casa más que salir a hacer cosas nuevas?, ¿Piensa que no vale para nada tal como está ahora?", dando como resultado positivo a un puntaje mayor o igual a tres.

Short Physical Performance Battery (SPPB) (7): Esta escala valora la capacidad funcional de los adultos mayores. Consta de tres partes que sirven para valorar la velocidad de la marcha, el equilibrio y la velocidad para poder levantarse y sentarse en una silla. A mayor puntaje se entiende una mayor funcionalidad física del paciente.

Índice de Barthel (8): Esta escala permite valorar la dependencia del paciente para realizar actividades básicas. Posee 10 preguntas acerca de la capacidad de realizar tareas cotidianas de forma independiente. A mayor puntaje, mayor independencia considerándose un puntaje de 100 independencia total para las actividades cotidianas y menor de 95 se traduciría en dependencia funcional.

Alcance funcional (9): Con ella, medimos el equilibrio del participante, en la cual le solicita ponerse de pie, con el hombro flexionado a 90 grados y estirando el brazo lo máximo posible sin levantar los pies del piso. Un puntaje menor a $10 \mathrm{~cm}$ se considera como potencial riesgo a caídas.

Luego de recolectar los datos, se analizaron mediante el programa STATA versión 14.0 calculando promedios $\mathrm{y}$ frecuencias, realizando un análisis bivariado en base a frecuencia de caídas, usando la técnica chi cuadrado para variables categóricas y $\mathrm{t}$ de Student para variables numéricas; finalmente se construyó un análisis de regresión de Poisson incluyendo en el modelo final a las variables significativas del análisis bivariado.

El presente trabajo de investigación cuenta con aprobación del comité de ética de la Universidad Científica del Sur con el código 377-2018-PRE15.

\section{RESULTADOS}

Se entrevistaron a 413 pacientes, de los cuales se excluyeron a 2 por falta de datos de las variables principales, quedando con un total de 411 pacientes. En la tabla 1 se describen las características de los participantes del estudio.

Se encontró una frecuencia de población femenina del 65,94\% (271 entrevistadas), en cuanto al grupo etario la mayoría de participantes tenían una edad entre los 70 a 80 años de edad (43,52\%). La mayor parte $(65,45 \%)$ vivía en una zona rural y el $82,48 \%$ eran analfabetos o tenían el colegio incompleto. El $77,62 \%$ vivía en compañía de alguien. La mayoría de participantes eran casados al momento de la aplicación del instrumento (57,91\%) seguido de divorciados/separados (28,71\%) solteros (10,71\%) y viudos $(2,68 \%)$. Al preguntárseles acerca de cómo consideraban su estado de salud la mayoría $(53,53 \%)$ lo consideraba como malo, seguido por regular en un $29,68 \%$.

Al preguntar acerca del consumo de fármacos, el 96,84\% consumía menos de 5 fármacos al día, en cuento a la presencia de comorbilidades el 45,94\% 
Tabla 1. Características de los participantes del estudio. $(\mathrm{n}=411)^{1}$

\begin{tabular}{|c|c|c|}
\hline Variables & $\mathbf{n}$ & $\%$ \\
\hline \multicolumn{3}{|l|}{ Datos socio-demográficos } \\
\hline \multicolumn{3}{|l|}{ Sexo } \\
\hline Femenino & 271 & 65,94 \\
\hline Masculino & 140 & 34,06 \\
\hline \multicolumn{3}{|l|}{ Edad en años } \\
\hline $60-70$ & 166 & 40,59 \\
\hline $70-80$ & 178 & 43,52 \\
\hline$>81$ & 65 & 15,89 \\
\hline \multicolumn{3}{|l|}{ Área de vivienda } \\
\hline Zona Urbana & 142 & 34,55 \\
\hline Zona Rural & 269 & 65,45 \\
\hline \multicolumn{3}{|l|}{ Grado de instrucción } \\
\hline Analfabeto/ colegio incompleto & 339 & 82,48 \\
\hline Colegio completo & 70 & 17,03 \\
\hline Técnico superior & 2 & 0,49 \\
\hline \multicolumn{3}{|l|}{ Vive sólo } \\
\hline No & 319 & 77,62 \\
\hline Sí & 92 & 22,38 \\
\hline \multicolumn{3}{|l|}{ Estado civil } \\
\hline Soltero (a) & 44 & 10,71 \\
\hline Casado (a) & 238 & 57,91 \\
\hline Viudo (a) & 11 & 2,68 \\
\hline Divorciado / separado (a) & 118 & 28,71 \\
\hline \multicolumn{3}{|l|}{ Autoreporte de salud } \\
\hline Excelente & 14 & 3,41 \\
\hline Muy bueno & 22 & 5,35 \\
\hline Bueno & 33 & 8,03 \\
\hline Regular & 122 & 29,68 \\
\hline Malo & 220 & 53,53 \\
\hline
\end{tabular}

Datos clínicos - Síndromes geriátricos

Polifarmacia

No

$398 \quad 96,84$

Sí

Presencia de comorbilidades

Ninguna

1

2 o mas

Antecedente de consumo de tabaco

No
Sí

Antecedente de consumo de alcohol

$\begin{array}{lll}\text { No } & 293 & 71,29 \\ \text { Sí } & 118 & 28,71\end{array}$

Antecedente de consumo de hoja de coca

$\begin{array}{lcc}\text { No } & 336 & 81,75 \\ \text { Sí } & 75 & 18,25\end{array}$

Dependencia funcional

No $\quad 129 \quad 31,39$

Sí $282 \quad 68,61$

Se siente frecuentemente triste o deprimido

$\begin{array}{lll}\text { No } & 255 & 62,04 \\ \text { Sí } & 156 & 37,96\end{array}$

Escala de Yesavage

$0-2 \quad 260 \quad 63,26$

3 a más $\quad 151 \quad 36,74$

Variables principales

Rendimiento Físico $^{2} \quad 7.29 \quad 3,09$

$\begin{array}{lll}\text { Alcance Funcional }^{2} & 19.8 \quad 6,75\end{array}$

Presencia de caídas

$\begin{array}{lll}\text { No } & 147 & 35,77\end{array}$

$\begin{array}{lll}\text { Sí } & 264 & 64,23\end{array}$

${ }^{1}$ Los valores absolutos pueden no sumar 411 debido a valores faltantes

(en ningún caso superaron el 10\% de la muestra)

${ }^{2}$ Media \pm Desviación Estándar

de la población presentaba 2 o más comorbilidades. En cuanto a consumo de tabaco el $88,08 \%$ de los participantes no fumaban, el $71,29 \%$ no consumía alcohol y el $81,75 \%$ no consumía hoja de coca.

Con respecto a la dependencia funcional, el 68,61\% tenía cierto nivel de dependencia funcional. El 64,23\% de los participantes afirmó haber sufrido al menos una caída en los últimos 12 meses, al preguntarle al participante si se sentía frecuentemente triste o deprimido solo el 37,96\% respondió que sí. En cuanto al test corto de Yesavage el 36,74\% de los participantes dio positivo para sintomatología depresiva. En cuanto a rendimiento físico, se presentó una media de 7,29 con una desviación estándar de 3,09 mientras que en alcance funcional daba una media de 19.8 con una desviación estándar de 64,23.

La tabla 2 muestra un análisis bivariado de caídas y covariables del estudio. Vemos que la mayoría de 
Asociación entre síndrome de caídas y síntomas depresivos en adultos mayores de once comunidades altoandinas.

Tabla 2. Análisis Bivariado de caídas y covariables del estudio $(\mathrm{n}=411)^{1}$

\begin{tabular}{|c|c|c|c|}
\hline Variables & Sin caídas & con caídas & Valor de $P$ \\
\hline \multicolumn{4}{|l|}{ Datos socio-demográficos } \\
\hline Sexo & & & 0,13 \\
\hline Femenino & $90(61,22)$ & $181(68,56)$ & \\
\hline Masculino & $57(38,78)$ & $83(31,44)$ & \\
\hline Edad en años & & & 0,014 \\
\hline $60-70$ & $65(44.22)$ & $101(38.55)$ & \\
\hline $71-80$ & $69(46.94)$ & $109(41.60)$ & \\
\hline$>81$ & $13(8.84)$ & $52(19.85)$ & \\
\hline Zona de vivienda & & & 0.057 \\
\hline Zona Urbana & $42(28,57)$ & $100(37,88)$ & \\
\hline Zona Rural & $105(71,43)$ & $164(62,12)$ & \\
\hline Grado de instrucción & & & 0,357 \\
\hline Analfabeto / colegio incompleto & $116(78,91)$ & $223(84,47)$ & \\
\hline Colegio Completo & $30(20,41)$ & $40(15,15)$ & \\
\hline Técnico Superior & $1(0,68)$ & $1(0,38)$ & \\
\hline Vive sólo & & & 0,82 \\
\hline No & $115(78,23)$ & $204(77,27)$ & \\
\hline Sí & $32(21,77)$ & $60(22,73)$ & \\
\hline Estado civil & & & 0,031 \\
\hline Soltero (a) & $23(15,65)$ & $21(7,95)$ & \\
\hline Casado (a) & $83(56,46)$ & $155(58,71)$ & \\
\hline Viudo (a) & $1(0,68)$ & $10(3,79)$ & \\
\hline Divorciado / separado (a) & $40(27,21)$ & $78(29,55)$ & \\
\hline Autoreporte de salud & & & 0,83 \\
\hline Excelente & $5(3,40)$ & $9(3,41)$ & \\
\hline Muy bueno & $8(5,44)$ & $14(5,30)$ & \\
\hline Bueno & $15(10,20)$ & $18(6,82)$ & \\
\hline Regular & $42(28,57)$ & $80(30,30)$ & \\
\hline Malo & $77(52,38)$ & $143(54,17)$ & \\
\hline \multicolumn{4}{|l|}{ Datos clínicos - Síndromes geriátricos } \\
\hline Polifarmacia & & & 0,006 \\
\hline No & $147(100,00)$ & $251(95,08)$ & \\
\hline Sí & $0(0,00)$ & $13(4,92)$ & \\
\hline Antecedente de consumo de tabaco & & & 0,038 \\
\hline No & $136(92,52)$ & $226(85,61)$ & \\
\hline Sí & $11(7,48)$ & $38(14,39)$ & \\
\hline Antecedente de consumo de alcohol & & & 0,0001 \\
\hline No & $122(82,99)$ & $171(64,77)$ & \\
\hline Sí & $25(17,01)$ & $93(35,23)$ & \\
\hline Antecedente de consumo de hoja de & & & 0,308 \\
\hline
\end{tabular}




\begin{tabular}{lccc} 
No & $124(84,35)$ & $212(80,30)$ & \\
$\quad$ Sí & $22(15,65)$ & $52(19,70)$ & \\
Comorbilidades & & & 0,167 \\
$\quad 0$ & $44(32,84)$ & $59(26,46)$ & \\
1 & $37(27,61)$ & $53(23,77)$ & \\
$\quad 2$ o más & $53(39,55)$ & $111(49,78)$ & \\
Variables principales & & & \\
Rendimiento físico ${ }^{2}$ & $9,22 \pm 2,94$ & $6,22 \pm 2,63$ & 0,0001 \\
Alcance funcional ${ }^{2}$ & $21,56 \pm 6,37$ & $18,87 \pm 6,78$ & 0,0001 \\
¿Se siente frecuentemente triste o deprimido? & & & 0,0001 \\
$\quad$ No & $112(76,19)$ & $143(54,17)$ & \\
$\quad$ Sí & $35(23,81)$ & $121(45,82)$ & \\
$\quad$ Escala de Yesavage & & & \\
$\quad 0-2$ & $124(84,35)$ & $136(51,52)$ & \\
$\quad>3$ & $23(15,65)$ & $128(48,48)$ & \\
\hline${ }^{1}$ Los valores absolutos pueden no sumar 411 debido a valores faltantes & & \\
(en ningún caso superaron el 10\% de la muestra) & & & \\
${ }^{2}$ Media \pm DE & & & \\
\end{tabular}

Tabla 3. Regresión de Poisson: Asociación entre caídas y depresión. $\mathrm{n}=411$ )

\begin{tabular}{lcc}
\hline Variables & PR crudo (IC 95\%) & PR ajustado $(\mathbf{I C 9 5 \% )})^{2}$ \\
Depresión & $1,62(1,27-2,06)$ & $1,40(1,06-1,86)$ \\
\hline${ }^{1}$ Prevalence ratio (Intervalo de Confianza al 95\%) \\
${ }^{2}$ Modelo de Regresión de Poisson ajustado por edad, estado civil, polifarmacia, consumo de alcohol, \\
consumo de tabaco, rendimiento físico y alcance funcional.
\end{tabular}

participantes con caídas fueron de sexo femenino $(68,56 \%)$ pero sin ser un dato estadísticamente significativo, en cuanto a edad el grupo etario con mayor porcentaje de caídas $(41,60 \%)$ fueron entre 71 a 80 años con datos estadísticamente significativo. Por otra parte, al hablar de estado civil, se vio en el grupo de participantes viudos que el 3,79\% habían sufrido caídas en comparación al $0,68 \%$ que no tenían historial de caídas en los últimos 12 meses, con datos estadísticamente significativos.

En lo referente a polifarmacia se vio que todos los pacientes que tenían antecedente de polifarmacia habían sufrido de caídas en los últimos 12 meses (4,92\% del total de participantes que habían sufrido caídas) con datos estadísticamente significativos. En cuanto a consumo de tabaco, se vio que los consumidores representaban el $14,39 \%$ de participantes que presentaron caídas. En el caso de los consumidores de alcohol, estos representaban un total del $35,23 \%$ de los participantes con presencia de caídas, datos estadísticamente significativos. En rendimiento físico (evaluado mediante la escala SPPB) se vio un promedio significativamente menor en los pacientes con caídas al igual que en alcance funcional. En cuanto al Autoreporte acerca de sintomatología depresiva los pacientes que respondieron que si fueron un $45,82 \%$ de los participantes con caídas. Finalmente, los pacientes con una escala de Yesavage de 3 o más puntos fueron un $48.48 \%$ del total de caídas, con datos estadísticamente significativos.

En la tabla 3 se muestra la asociación entre caídas y depresión mediante la regresión de Poisson en el cual se evidencia un PR crudo (con intervalo de confianza en 95\%) de 1,62 y un PR ajustado de 1,40

\section{DISCUSIÓN}

El 2010, en Canadá se realizó un metaanálisis en el cual se encontró que la sintomatología depresiva incrementaba en un 1,62 veces la posibilidad de caídas 
Asociación entre síndrome de caídas y síntomas depresivos en adultos mayores de once comunidades altoandinas.

en personas adultas mayores (10); esto es similar a lo encontrado en nuestro análisis en el cual podemos ver que existe una asociación entre los síntomas depresivos y las caídas, siendo este un factor de riesgo. En relación al estudio ya citado, se encontró asociación entre caídas y el sexo del participante, encontrándose que había 1,30 veces más riesgo de caídas en el sexo femenino, al compararse con nuestra investigación se pudo apreciar que no era un factor significante en nuestra población. Este hallazgo podría deberse a que, en ambos sexos, la actividad física a expensas de necesidad de continuar trabajando sería más frecuente.

En Estados Unidos, se realizó un estudio en el cual se buscaron predictores de caídas en adultos mayores residentes de asilos, se encontró que la depresión aumentaba en 1,9 veces la posibilidad de sufrir una caída, además se evidenció que la presencia de escaleras en estos centros podía llegar a aumentar hasta en 6 veces la posibilidad de caídas (11).

Bajo el mismo contexto, en Francia, se midió depresión mediante el GDS-15 encontrándose también que la depresión aumentaba el riesgo de caídas (12). En Australia se realizó un estudio similar en el cual menciona que la presencia de sintomatología depresiva (medida con el GDS) aumenta en 1,64 la posibilidad de caídas (13).

En el Perú, el año 2017 se realizó un estudio para determinar los factores asociados a caídas a nivel hospitalario, en el cual los autores demostraron que el sexo femenino presenta alto riesgo de caídas $(14,15)$; al igual que nuestro estudio, encontró que tanto la zona de vivienda como el grado de instrucción fueron factores asociados.

Existe escasa evidencia de asociación entre caídas y depresión en el contexto de adultos mayores residentes en comunidades rurales o urbanas de los Andes u otras zonas de altura, que, por temas de adaptación climatológica, barométrica, social, de acceso a servicios de salud y otros factores, podrán tener factores de riesgo distintos y necesidades de salud diferenciadas en comparación con otras realidades.

En conclusión, nuestro estudio arrojó que los adultos mayores con sintomatología depresiva (según test de Yesavage) tiene 1,62 veces más posibilidades de presentar caídas que las personas que no presentaron depresión y que, independientemente de otros factores que tienen cierto grado de asociación, hay 1,40 veces más chance de sufrir de caídas. Además, factores asociados con caídas como el rendimiento físico, el alcance funcional, el estado civil, la edad, la polifarmacia, los hábitos nocivos, además de la depresión y síntomas depresivos, deberían ser tomados en cuenta en la valoración integral del adulto mayor en riesgo de caídas.

Conflictos de interés: los autores declaran no tenerlos. Agradecimientos: a los docentes de la Universidad Científica del Sur, por el apoyo para que el presente estudio se cristalice.

\section{Correspondencia:}

Lucía Casahuaman-Orellana

Correo electrónico: jimenacasahuaman@gmail.com

\section{REFERENCIAS BIBLIOGRÁFICAS}

1. Osoba MY, Rao AK, Agrawal SK, Lalwani AK. Balance and gait in the elderly: A contemporary review. Laryngoscope Investigative Otolaryngology.2019; 4(1): 143-153. doi: https://doi.org/10.1002/lio2.252

2. Galván-Parra Y, Moreno-Castillo Y, GonzálesPedraza. A. El síndrome de caídas y la calidad de vida relacionada con la salud en el adulto mayor. Archivos en Medicina Familiar. 2009; 12(1): 17-24.

3. Brandão DJ, Fontenelle LF, da Silva SA, Menezes PR, Pastor-Valero M. Depression and excess mortality in the elderly living in low-and middle-income countries: Systematic review and meta-analysis. Int J Geriatr Psychiatry. 2019;34(1):22-30. doi: 10.1002/ gps.5008

4. Instituto Nacional de Estadística e Informática. Informe técnico estadísticos de población adulta mayor en el Perú. Lima: Instituto Nacional de Estadística e Informática; 2018 (Fecha de acceso 20 de Enero 2019) Disponible en: https://www.inei.gob. pe/media/MenuRecursivo/boletines/informe-tecnicosituacion-de-la-poblacionadulta-mayor_dic2018.pdf

5. Urrunaga-Pastor D, Runzer-Colmenares F, Arones T, Meza-Cordero R, Taipe-Guizado S, Guralnik $\mathrm{J}$, et al. Factors associated with poor physical performance in older adults of 11 Peruvian high Andean communities. F1000Res. 2019;8:59. doi: 10.12688/f1000research.17513.1.

6. Gómez-Angulo, C, Campo-Arias, A. Escala de Yesavage para Depresión Geriátrica (GDS-15 y GDS-5): estudio de la consistencia interna y estructura factorial. Universitas Psychologica. 2011;10(3):735743

7. Estela-Ayamamani D, Espinoza-Figueroa J, Columbus-Morales M, Runzer-Colmenares F, Parodi J, Mayta-Tristán P. Rendimiento físico de adultos mayores residentes en zonas rurales a nivel del mar 
y a gran altitud en Perú. Revista Española de Geriatría y Gerontología. 2015;50(2):56-61. DOI: 10.1016/j. regg.2014.11.001

8. Wade D, Collin C. The Barthel ADL Index: A standard measure of physical disability? Int Disabil Stud. 1988;10(2):64-67.

9. Cerda L. Manejo del trastorno de marcha del adulto mayor. Revista Médica Clínica Las Condes. 2014;25(2):265-275.

10. Iaboni A, Flint A. The complex interplay of depression and falls in older adults: A clinical review. Am J Geriatr Psychiatry. 2013;21(5):484-92. doi: 10.1016/j.jagp.2013.01.008

11. Byers AL, Sheeran T, Mlodzianowski AE, Meyers BS, Nassisi P, Bruce ML. Depression and risk for adverse falls in older home health care patients. Res Gerontol Nurs. 2008;1(4):245-51. doi: 10.3928/19404921-20081001-03

12. Turcu A, Toubin S, Mourey F, D'Athis P, Manckoundia P, Pfitzenmeyer P. Falls and depression in older people. Gerontology. 2004;50(5):303-8.
13. Kvelde T, Lord S, Close J, Reppermund S, Kochan N, Sachdev P, et al. Depressive symptoms increase fall risk in older people, independent of antidepressant use, and reduced executive and physical functioning. Arch Gerontol Geriatr. 2015;60(1):190-5. doi: 10.1016/j.archger.2014.09.00

14. Espinoza A, Tinocco G. Relación entre riesgo de caídas y principales factores sociodemográficos en pacientes de geriatría "Hospital Félix Mayorca Soto" Tarma-Junio 2016. Tesis para Licenciatura. Lima, Perú: Universidad Católica Sedes Sapientiae; 2017.

15. Theou O, Brothers T, Mitnitski A, Rockwood K. Operationalization of frailty using eight commonly used scales and comparison of their ability to predict all-cause mortality. J Am Geriatr Soc. 2013; 61(9):1537-51. doi: 10.1111/jgs. 12420

Recibido: 31/01/2019 Aceptado: 24/03/2019 